\title{
An LSTM-Based Approach to Predict Stock Price Movement for IT Sector Companies
}

\author{
Shruthi Komarla Rammurthy, Global Academy of Technology, Bengaluru, India \\ Sagar B. Patil, School of Management Studies and Research, KLE Technological University, Hubli, India \\ (iD https://orcid.org/0000-0002-3797-9449
}

\begin{abstract}
A stock market is an aggregation of buyers and sellers where issuance, buying, and selling of stocks happen. Predicting stock price is a significant concern due to volatility. Historic stock price and historic price data reveal the effect of such factors. Since stock data is time series and prediction can be made accurately with time series forecasting model. LSTM (long short-term memory) model, a particular kind of RNN (recurrent neural network), based on time series forecasting, is used to predict stock price. LSTM doesn't have long-term dependencies because of its distinctive structure. The study focuses on major IT firms considering the company's low and high prices. But, mid-price, which is a mean of the low and close price, is considered for the prediction. LSTM-based methodology employing mid-price is effective in predicting values compared to other attributes and accuracy of prediction using the LSTM model. The authors conclude with the present model is more efficient in stock price prediction with a decrease in mean square error.
\end{abstract}

\section{KEYWORDS}

IT Companies, LSTM, Prediction, RNN, Stock Market Returns, Time Series

\section{INTRODUCTION}

The stock market provides the platform for the investors to buy and sell investments avenues, especially equities. A majority of people are looking forward to find a model that guides in stock market. The stock market works through a series of interactions. In India, it is NSE (National Stock Exchange) and BSE (Bombay Stock Exchange). Prediction of stock prices has gain major attention in academia and industry as well. This attention towards prediction is majorly due to market volatility and less awareness of the companies among investors. Most of the Machine Learning algorithms help in analyzing stock market and predicting the prices by considering various factors. Predicting stock prices is a complex task as it involves various factors including rational and irrational, physical and psychological, political, market rumors, interest rates, investor mood and so on (Ghosh et al., 2019). These factors can be handled by Artificial Intelligence which considers the past data, social sentiment analysis and company's net growth to predict stock prices. Deep Neural Networks and RNN can handle multivariate time series data very well. The training is done on these networks by giving company's past data and predicts stock prices as output of the model.

There are so many other models available in order to predict stock prices like regression, ARIMA, weighted moving average, CARIMA etc. Here, authors employed LSTM along with linear regression to predict stock prices. LSTM's provide a promising solution for time series data. LSTM can retain 
the information for a long period of time which provides us more accurate results. Since, stock price of a company changes time to time and even depends on other intrinsic and extrinsic factors, it is very difficult to predict stock price. In Indian context companies enter secondary markets through primary markets i.e., thorough IPO's they issue their shares that are later traded on to registered stock exchanges. Bombay Stock Exchange is one of the oldest stock exchanges of India with over 5000 companies listed. Once shares are listed in secondary market then their price is determined by market forces like demand and supply. The price of company share moves up, if more people want to buy the shares than selling it and vice versa. This variation in the supply and demand of company's stock prices depends on various factors like intrinsic and extrinsic factors. Some of the intrinsic factors are earnings of the company, liabilities, demand stability, competition in market, technically advanced assembly line, surplus cash for adverse situations, stakes in raw material supplier and finished product distributors etc. Those factors that are beyond the control of the company such as crude oil price, dollar exchange rate, political stability, government policy decision etc. refers to extrinsic attribute (Ghosh et al., 2019).

Stock market is a nonlinear time series data which is highly fluctuating (Ma et al., 2018). A time series can be used to obtain status of some activity for a set of data measured for a certain time period (Hamzaebi et al., 2009). Linear models like AR, ARMA, ARIMA (Menon et al., 2016; Zhang, 2003) forecast the stock market using moving average and auto regression. These models when trained work differently for different companies which is a serious concern. This makes prediction of stock price very difficult. Many researchers have tried using the historical stock prices as the basis for time series analysis to forecast future stock prices (Ghosh et al., 2019). In this paper, various attributes of a company are compared to predicting stock price. A special RNN, LSTM, a deep neural network which can learn from past experience and can provide accurate results is been used. Results shows how various attributes contribute towards predicting stock price.

\section{BACKGROUND}

\subsection{Indian Stock Market Overview}

The trading in Indian stock market happens in two stock exchanges- BSE (Bombay Stock Exchange) and NSE (National Stock Exchange). For any company to sell its shares or to be a part of trading, their credentials have to be registered in stock exchange. Authorities of stock exchange verify the identity of the company, their financial stability and even other facets of the company. The trading procedure, trading hours and settlement process is almost similar in both the Stock Exchanges. The trading procedure for any company would start with the selection of broker, where registered brokers would help in buying and selling of shares. Then any investor, who wishes to be a part of trading, has to open a Demat (Dematerialized) account which can preserve balances of investor and convey investor periodically about their holdings. Once the account is opened the investor can place buy and sell orders through registered brokers. Upon the instructions of investor, broker executes the order and facilitates the trade.

The two main predominant market indices for BSE and NSE are Nifty and Sensex. These indicators give an idea whether stock prices of various companies have increased or decreased. These can also be used to compare various companies and can be used as a reflector of investor's sentiments. Since last few years, internet based technologies have played a very important role in Indian Stock market. Facebook, Twitter and other social media has impact on stocks prices of a company (Bhardwaja et al., 2015). 
Figure 1. Recurrent Neural Networks (RNN) are the type of Neural Networks that allow previous output to be taken as input for the next state by having hidden layers

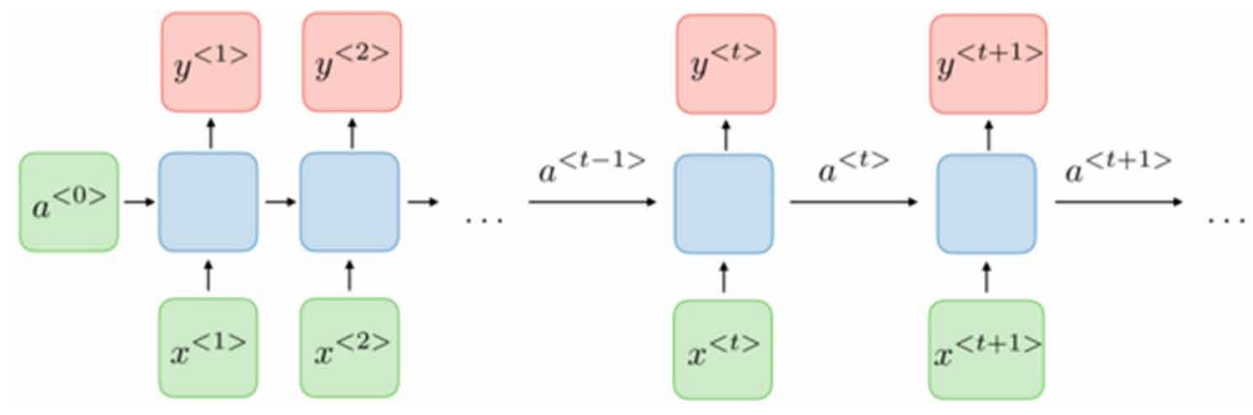

\subsection{Recurrent Neural Network}

Recurrent Neural Networks (RNN) are the type of Neural Networks that allow previous output to be taken as input for the next state by having hidden layers as shown in Figure 1. This can be treated as chain of repeated neural networks. This would reflect real world scenario. Let us take an example:

In RNN, initially it takes the input $\mathrm{x}^{1}$ and gives the output $\mathrm{y}^{1}$ by using activation function. In later stages, it takes two inputs- $x^{2}$ and previous output i.e. $y^{1}$ to generate the output $y^{2}$ and the same continues. This information is passed from one stage of the network to other stage through this loop. Advantage of RNN is it can consider historical information. So, it is good to use RNN for predicting stock market as prediction requires historical information of various companies. But, the major drawback in RNN is: If the information is too old, it cannot predict the correct instance accurately (Ghosh et al., 2019). This is resolved by the special kind of RNN, LSTM network.

\subsection{LSTM Networks}

LSTM- Long Short Term Memory Networks are the typical kind of RNN which was developed by Hochreiter and Schmidhuber in 1997 (1997). Long term dependency problem is resolved in LSTM network by remembering the long term information. Figure 2 represent architecture of LSTM cell (Ma et al., 2018). The architecture of LSTM is bit different than compared to other networks that include memory block or cells to store previous data.

In the Figure 2:

$\mathrm{C}_{\mathrm{t}-1}$ - old cells state

$\mathrm{C}_{\mathrm{t}}$ - present cell state

$\mathrm{h}_{\mathrm{t}-1}-$ output of previous cell

$\mathrm{h}_{\mathrm{t}}-$ output of previous cell

$i_{t}-$ input gate layer

$\mathrm{f}_{\mathrm{t}}$ - forget gate layer

$\mathrm{O}_{\mathrm{t}}-$ output sigmoid gate layer

\subsection{Working of LSTM}

The main part of LSTM is cell state which is represented by horizontal line at the top of the diagram. Any information can be added or removed in LSTM through these gates. These gates are structured using sigmoid function and point-wise multiplication operation. In the next step which is a combination of tanh and sigmoid layer helps to add old information to new information. Then it updates the new cell state. Finally, output is based on cell state, but will be a filtered version. First, when the sigmoid 


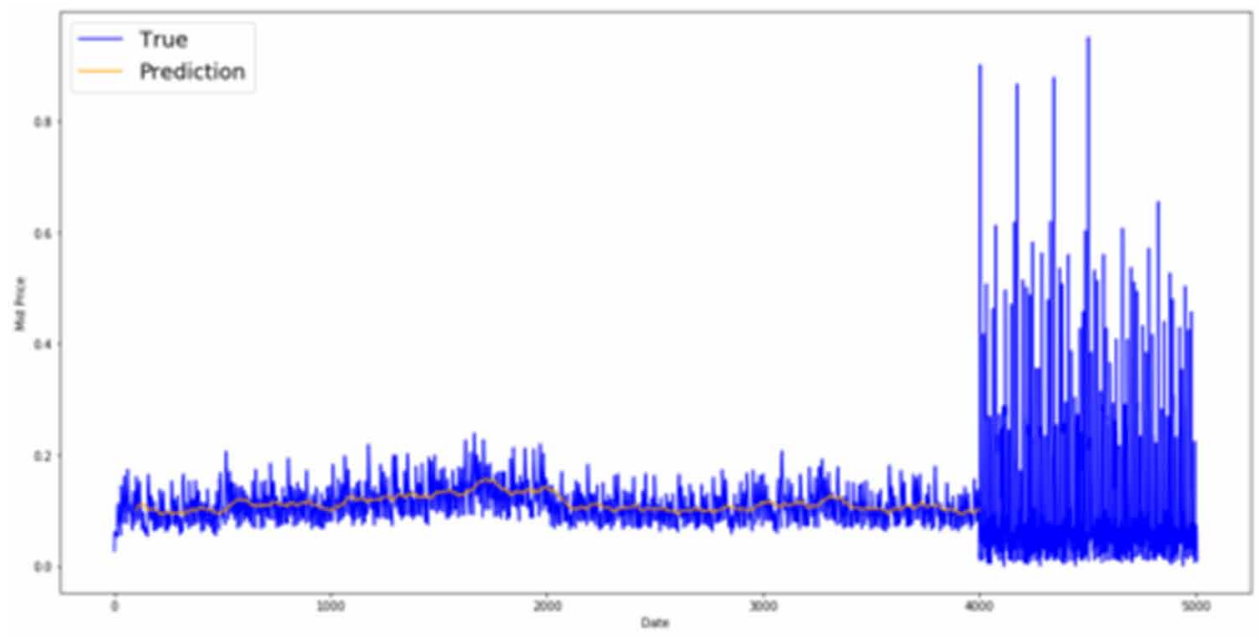

layer is run it decides the parts of cell state to be given as output. Then, these parts of the cell state are given to tanh and is then multiplied by output of the sigmoid gate, so that needed information is given as output.

\section{RELATED SURVEYS}

Prediction of stock prices is being major attraction, there is a lot of research being done using various machine learning algorithms. Macro and Micro factors have been considered for prediction. Stock market analysis can be grouped into three categories: a) Fundamental analysis b) Technical Analysis c) Sentimental Analysis. Fundamental Analysis tries to estimate value of stock based on factors like current financial stability of the company, prevalent economic environment and so on. Technical Analysis helps to figure out stock price based on recent trading movements of the company. Sentiment Analysis attempt to measure market in terms of attitude of investors. Many of Machine learning algorithms uses time series data of companies to do prediction like linear regression, multiple regression, classifiers like SVM, random forest or Neural Networks like CNN, RNN or so on.

Authors in (Ghosh et al., 2019) have analyzed stock prediction using company's net growth and historical data employing LSTM algorithm. In this paper, they predicted stock price and company's future growth in various time slots and came to conclusion that their framework gives the best prediction for 3 months.

Authors in (Ding \& Qin, 2019) used LSTM to predict stock returns for china market. China's historical data was categorized as 30 days long sequences with 10 learning features and 3 day earn rate labeling. The training of the model was done using 900000 sequences and testing was done on other 311361 sequences. The dataset that authors used described performance of stock with sequence learning features like closing price and trading volume on each day basis. They could get more accuracy than compared to other algorithms.

Predicting stock prices using sentiment analysis was done by authors in (Bhardwaja et al., 2015) using data from Sensex and Nifty. In this paper, authors collected the data online from Nifty and Sensex using Beautiful Soup. Later they use Text mining and Natural Language Processing to do Sentiment Analysis. This helped to maintain economical balance of share market. This helped investors to possess knowledge on making better investment on desired shares. 
Historical data of any company contain many details. Extracting essential features from them is a tedious task. This task is accomplished using LSTM. Authors in (Qiu et al., 2020) have used Wavelet Transform which is based on attention mechanism and LSTM, to extract important features and train the same. The prediction model is established to predict stock price. Results from experiments in (Qiu et al., 2020) have shown attention based LSTM performs better than other 3 models.

RNN, a recurrent neural network model is used extensively for time series applications. But the major drawback of this model is its long term dependency issues which arise from its basic structure. So, in order to overcome the long term dependency, Vanilla LSTM was proposed which is the most commonly used LSTM. Author in (Sherstinsky, 2020) has explained clearly with equations moving from RNN to LSTM. Author has used Albeit unconventional notation to express and ease understanding of LSTM networks.

In this article, LSTM, a special RNN model is used to predict stock prices using Fundamental Analysis. LSTM model is selected for prediction as it out performs RNN especially in avoiding long term dependencies. Important characteristics are extracted in previous data which is a needed requirement in prediction of stock price. Given, past data of a company, LSTM can learn the data and can analyze the pattern of stock price movement. This learning can help predicting the stock more accurately.

\section{PROPOSED METHODOLOGY}

The following modules have been identified to perform the analysis of various parameters to do stock prediction.

1. Data Collection - Infosys data is collected from thehttps://www.bseindia.com/markets/equity/ EQReports/StockPrcHistori.aspx expandable $=6 \&$ scripcode $=512289 \&$ flag $=$ sp $\&$ Submit $=$ G. This dataset contains the parameters like Open price, Close Price, High price and Low price, WAP and other characteristics. In the analysis, above parameters are considered for stock prediction using LSTM, a RNN model. Nearly 250 months dataset is taken for analysis. Dataset is divided into training data set and test data set. Training data set is used to train the model and Test data set is used for prediction.

2. Data Preprocessing- The dataset collected is the raw data which can be inconsistent, incomplete and cannot be used to represent in efficient format. Data preprocessing involves methods to convert the raw data into useful and efficient format. The first step is to normalize the data. Data normalization is generally done when attributes contain varied range of values. Normalization coverts these values to common scale without distorting. Minmax Scalar function is used to normalize the data. But Minmax scalar function converts the data in the range of 0 to 1 . So the data is normalized by splitting the data into window size that suits the dataset.

3. Training Module - LSTM (Long Term Short Module) work excellently for time series data. This RNN model learns network considering past data. LSTM a special kind of RNN develops a model by using data generator and data augmentation. Model considers 3 layers of LSTM's and linear regression. Three layers of LSTM are encapsulated in MultiRNNCell in Tensorflow. This model is trained for past data by dividing data into batches. Output is again fed into neural network by finding loss between predicted stock prices and actual ones. Finally, model is optimized using Adam optimizer. Model is trained for train data and trained model does prediction on test data.

4. Prediction - The model tries to predict stock price by considering high price which is an average of company's low and high price. Prediction is done using dynamic_rnn function which uses prediction model and train dataset. Obtained result is sent to Regression layer. Regression layer, a model that finds least mean square error is used. This process is repeated for fixed number of epochs. During this process, model learns stock price movements, and tries to predict almost accurately. 


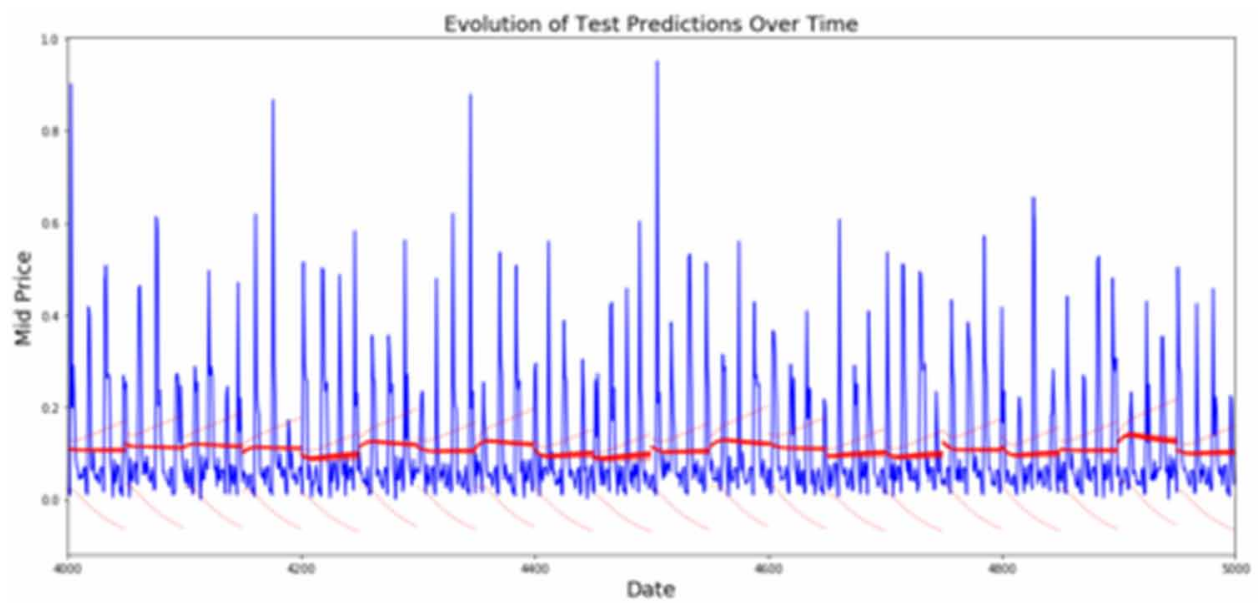

Best Test Predictions Over Time

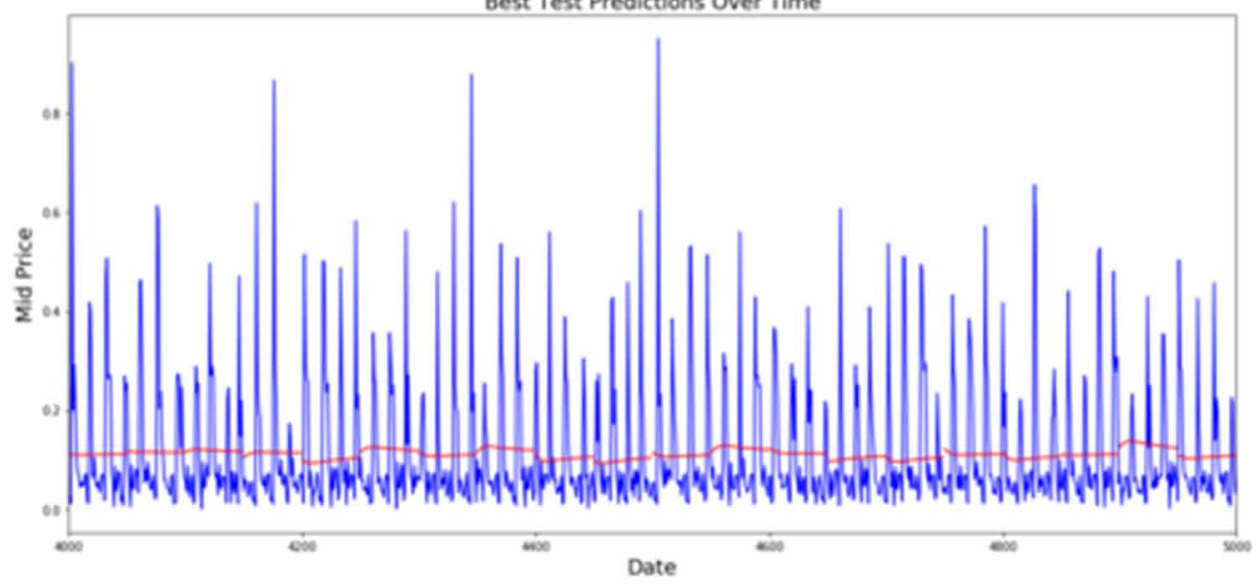

This model has shown a good prediction than compared to standard averaging. The analysis is done for various companies under IT sector and analysis shows how LSTM model performs better by considering mid price, which is an average of low and high price of a company's stock price (Figure 3 ).

\section{EXPERIMENTAL RESULTS AND DISCUSSION}

The experiment is conducted for various companies using mid price which is an average of low price and high price. Here, data obtained is first normalized using MinMaxScalar Function. Then, Standard Averaging is performed on smoothened data. The results obtained from Standard Averaging are compared against LSTM model. In LSTM, a data generator is first implemented which has a method called unroll batches which outputs a set of num_unrollings, which determines the number of time steps you look into future. In this model, a num_ unrollings of 50 is used and a batch size of 500 is used. Finally MultiRNN Cell in TensorFlow is used to combine three LSTM layers. An epoch size of 30 is used in the experiment. Results show that MSE is reduced on every epoch which clearly indicates model is learning and is able to predict stock price movement. In this paper, the results of Standard Averaging and LSTM are taken for four different companies under IT sector. The results of MSE for various companies and epochs are also presented. 


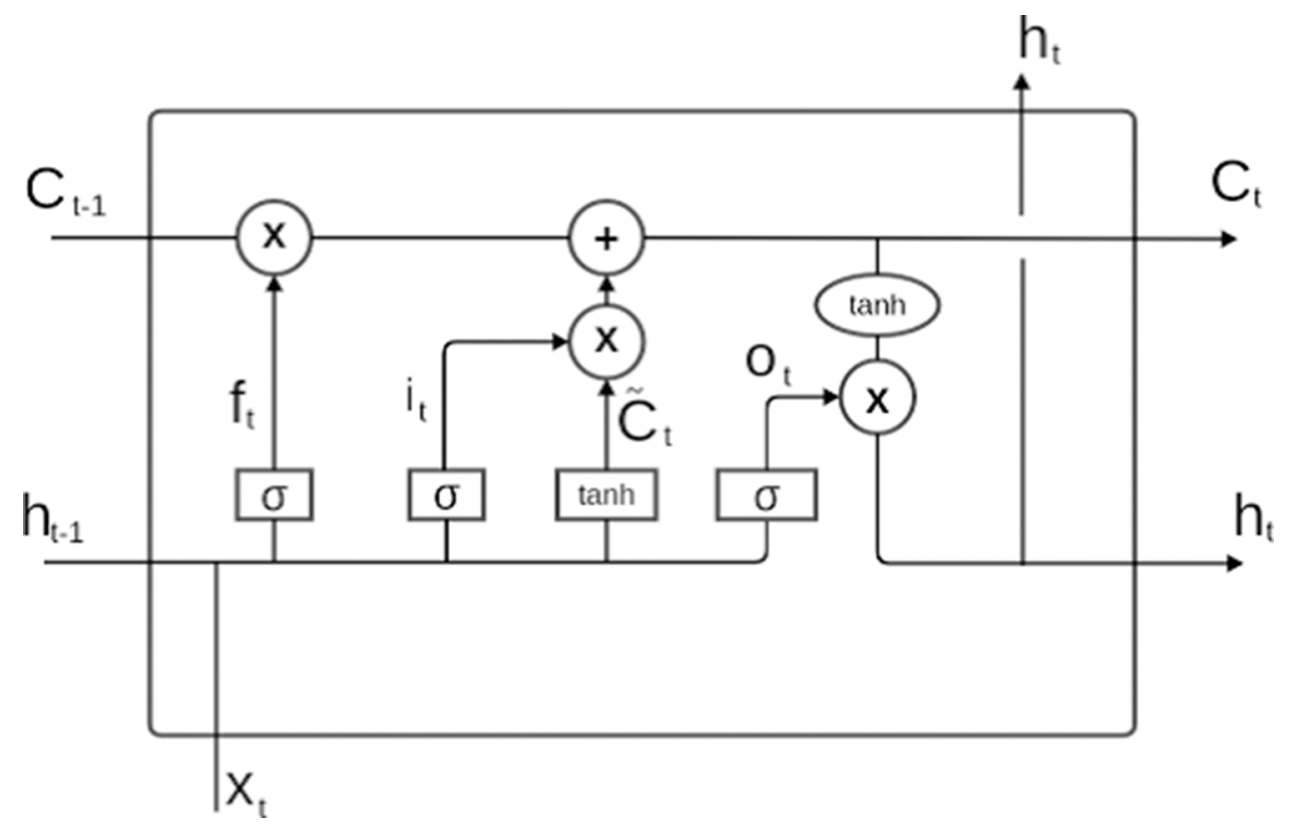

Figure 5. LSTM model with 30 epochs gave better accuracy than compared to Standard Averaging

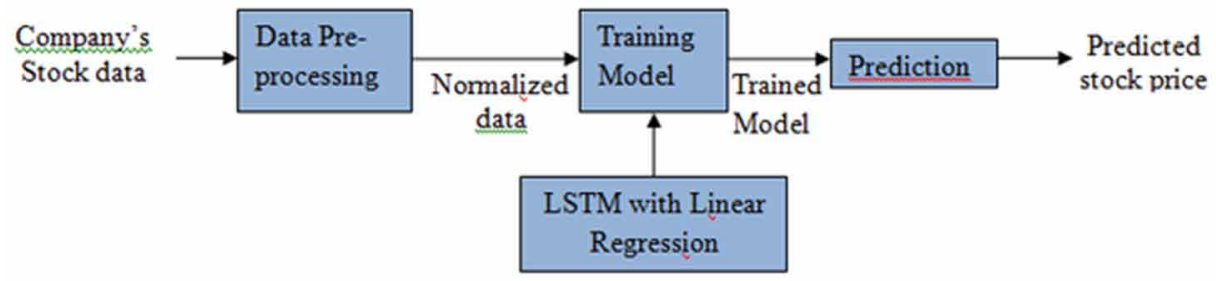

\subsection{Results of Infosys Stock Price Prediction}

Here, 3800 samples of Infosys stock prices are considered in this model. From these samples 3000 samples were used for the training purpose and other 800 samples are used for testing purpose. Standard Averaging method and LSTM model were employed on these samples. From the graphs plotted, as shown in Figure 4 and 5, LSTM model with 30 epochs gave better accuracy than compared to Standard Averaging.

\subsection{Results of TCS Stock Price Prediction}

Historical stock prices of TCS were considered for the prediction. Out of 3800 samples, 3000 samples were used for training purpose and the rest was used for testing. The results were improving over the epochs as mean square error was decreasing. Accuracy of the prediction was improved as compared to Standard Averaging. The results are shown in Figure 6 and 7. 
Figure 6. Accuracy of the prediction was improved as compared to Standard Averaging

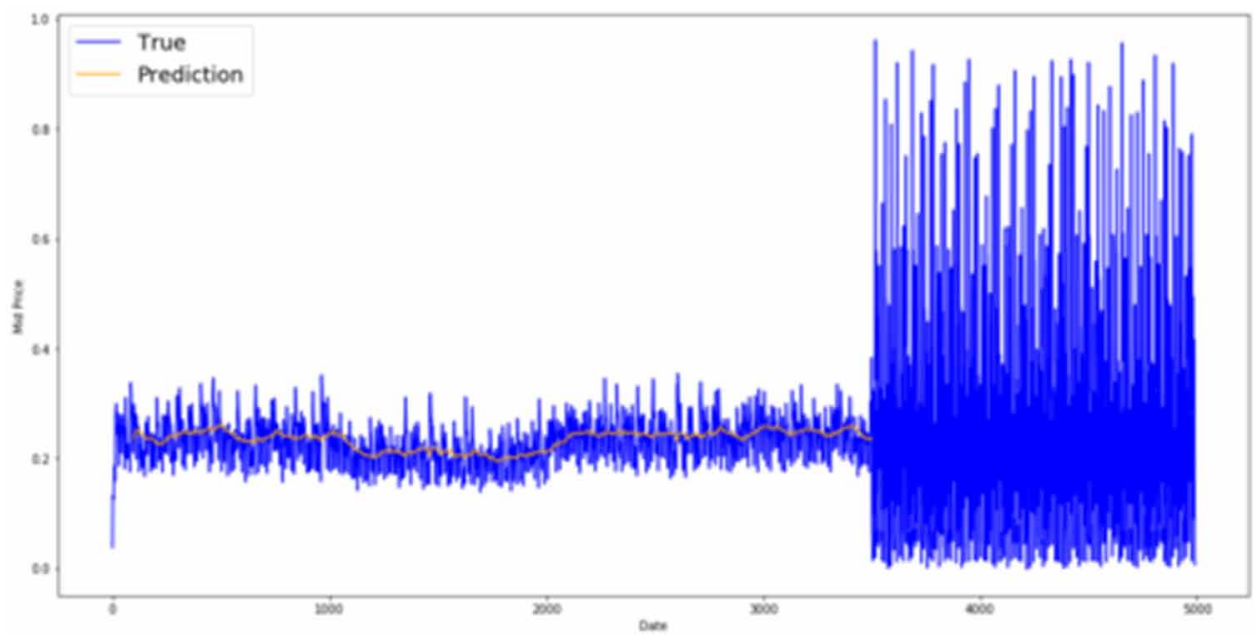

Figure 7. Comparison
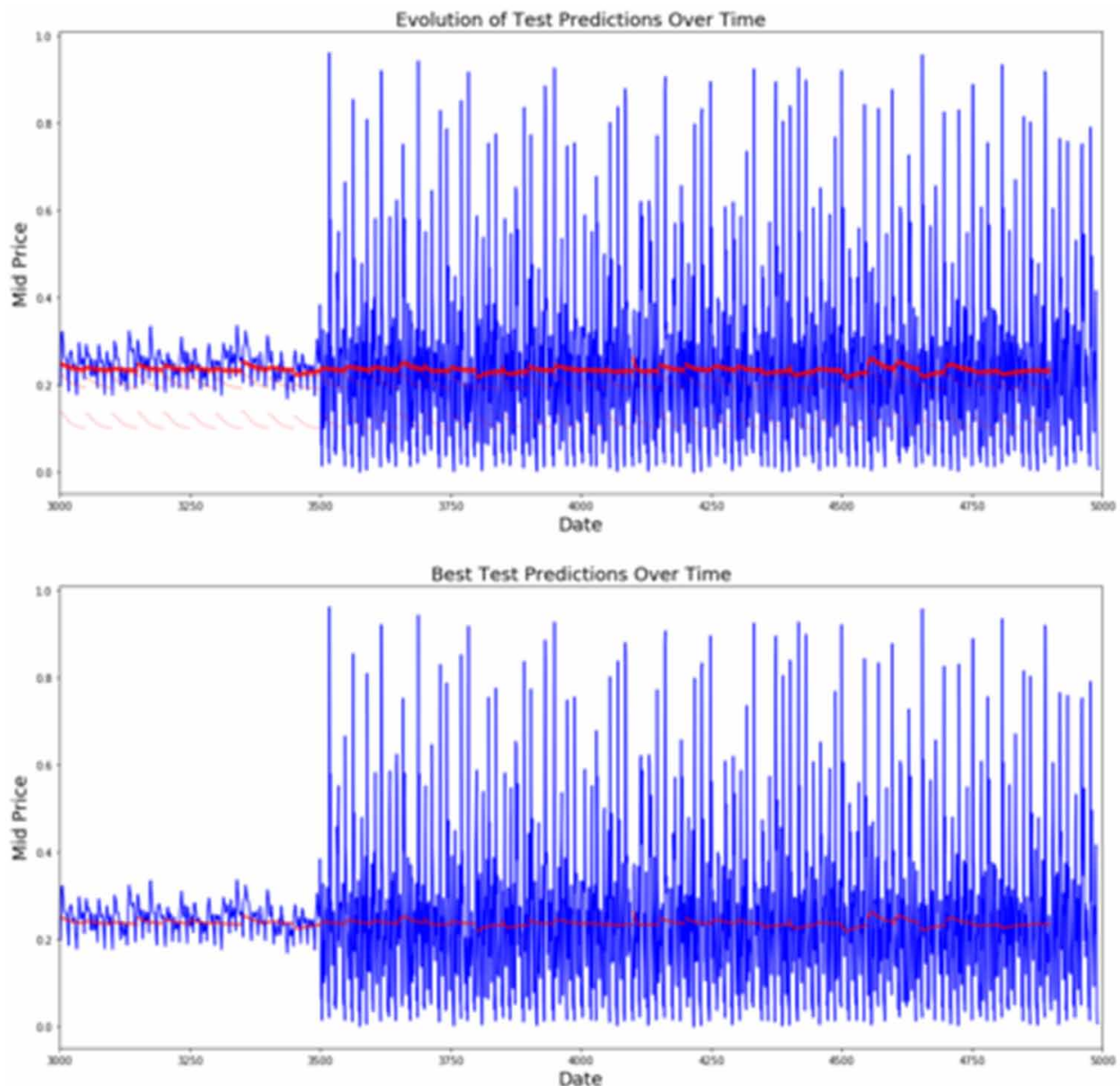
Figure 8. Predictions of the 2800 samples that were used in training along with other samples that were used for testing

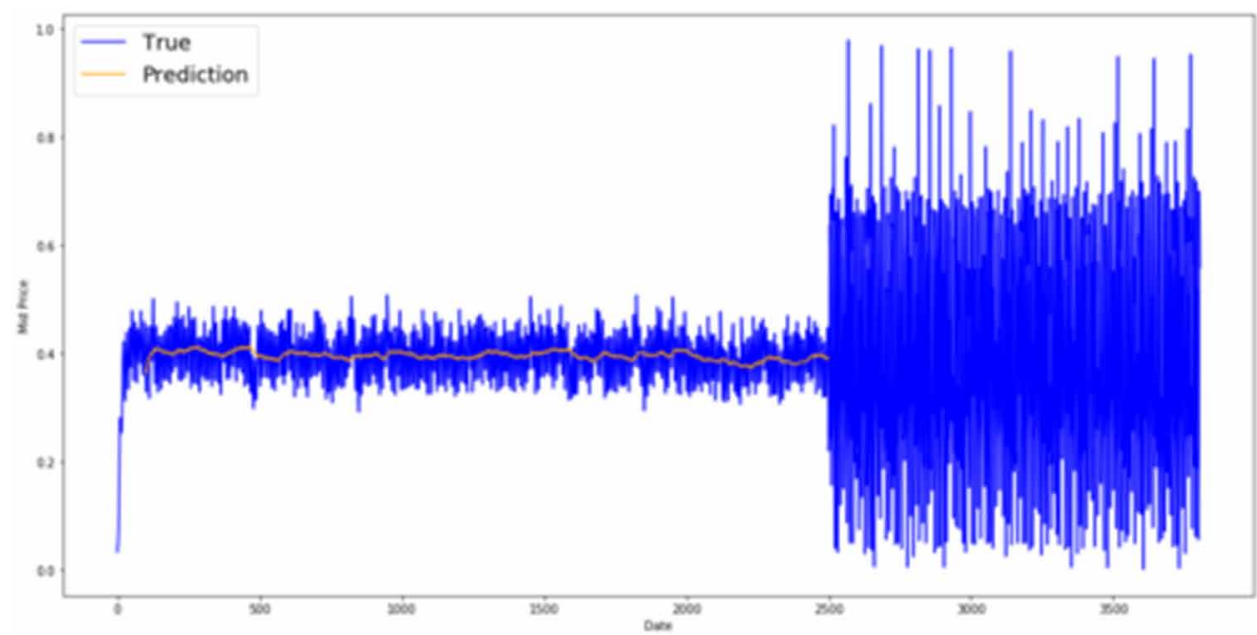

Figure 9. Comparison

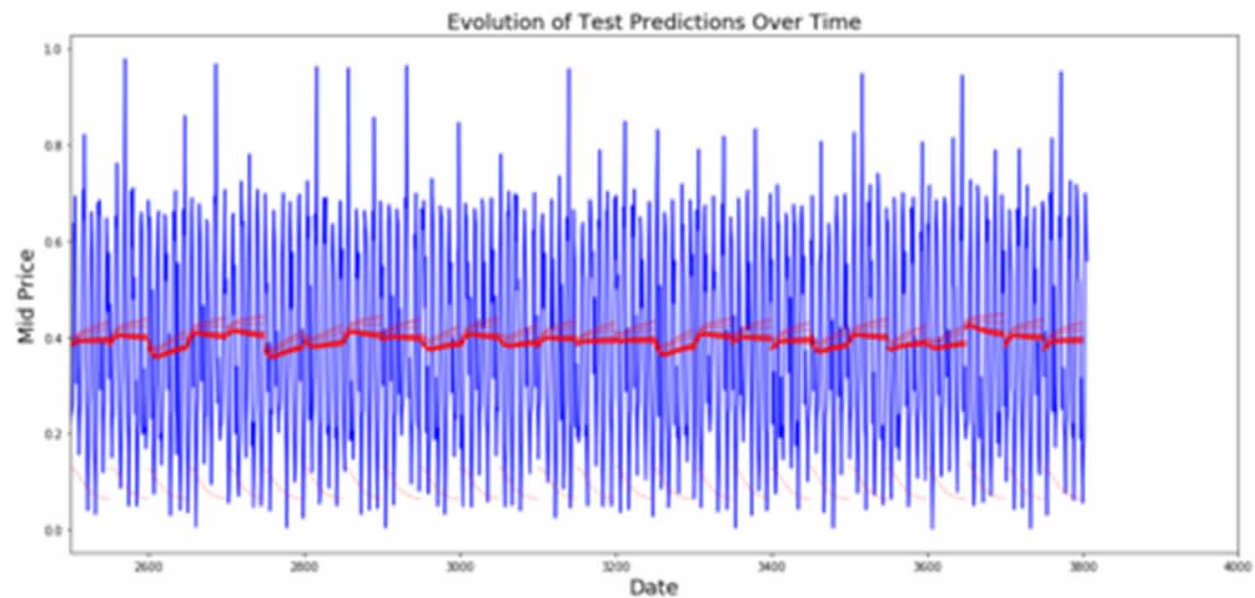

Best Test Predictions Over Time

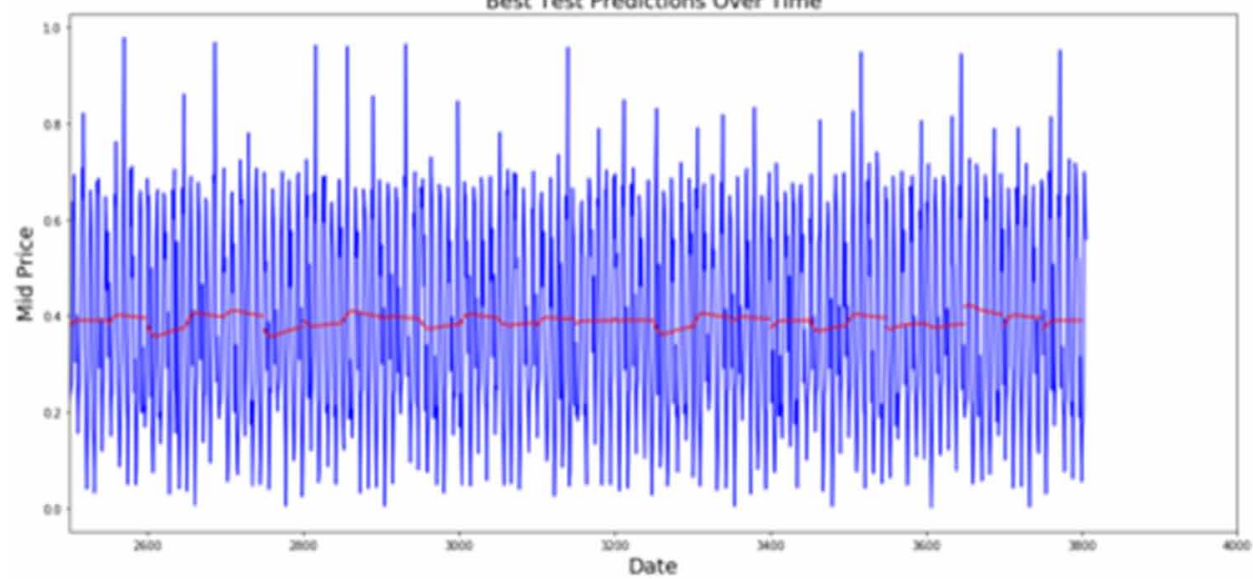


International Journal of Cognitive Informatics and Natural Intelligence

Volume 15 • Issue 4 • January-March 2021

Figure 10. Results of the prediction

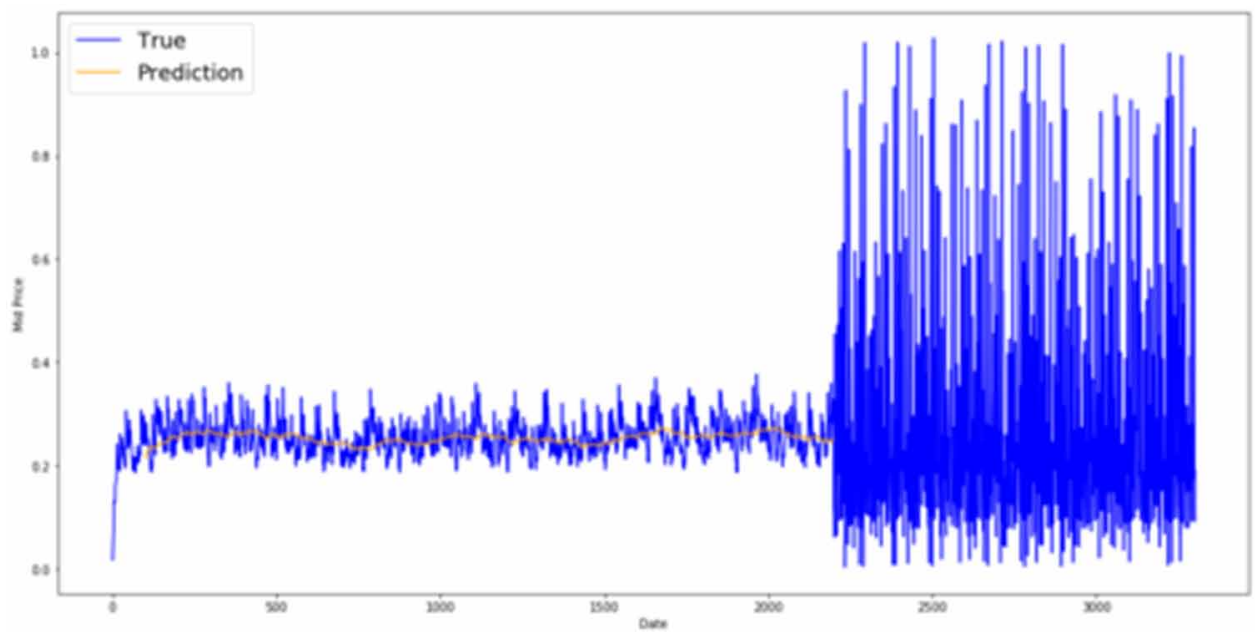

Figure 11. Comparison
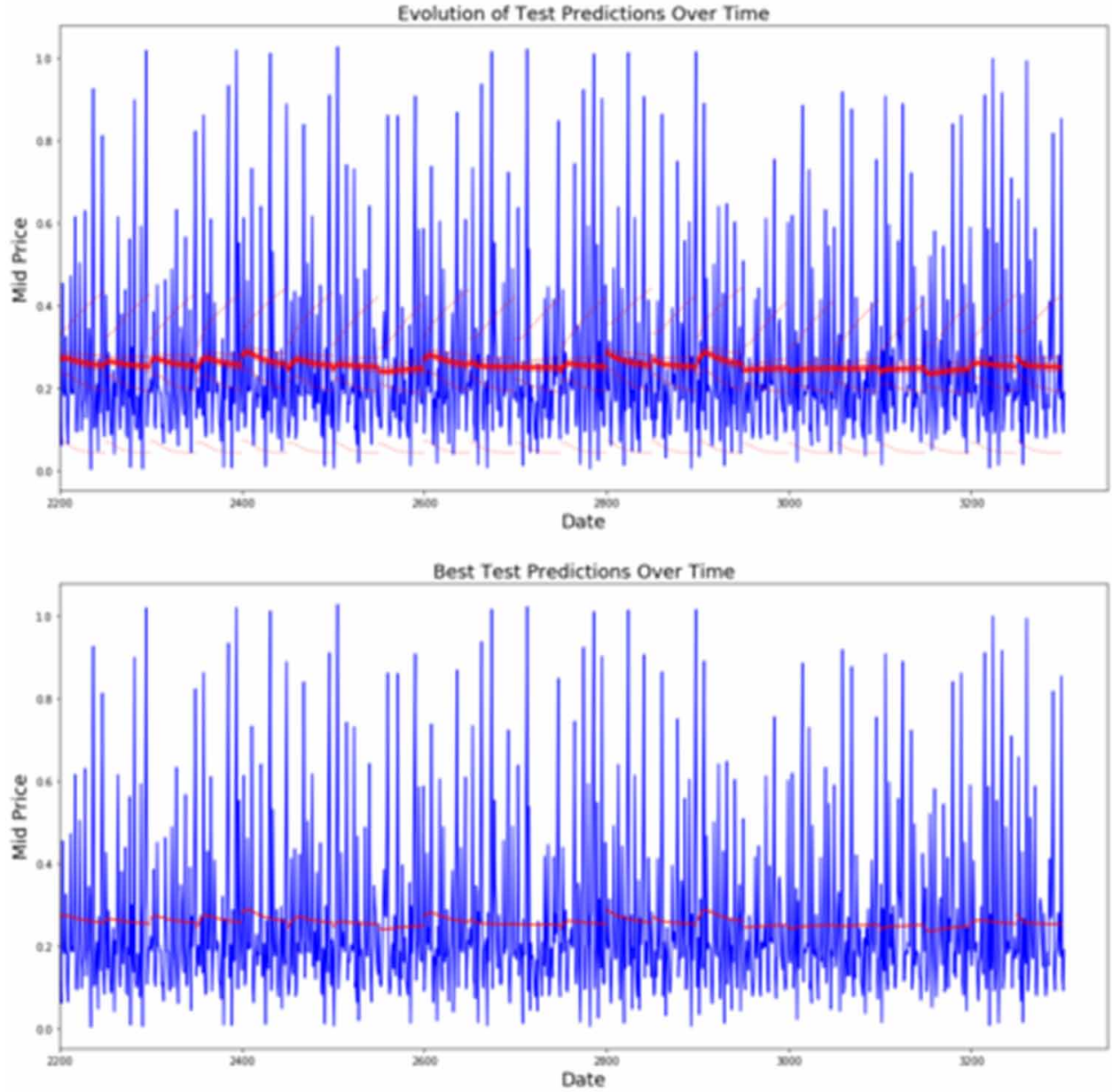
Table 1. Epoch readings

\begin{tabular}{|l|l|l|}
\hline Companies/Epoch & $\begin{array}{l}\text { Epoch 1 } \\
\text { MSE }\end{array}$ & $\begin{array}{l}\text { Epoch 30 } \\
\text { MSE }\end{array}$ \\
\hline Infosys & 0.0243 & 0.0136 \\
\hline TCS & 0.06757 & 0.02315 \\
\hline Tech Mahindra & 0.04039 & 0.01973 \\
\hline WIPRO & 0.01810 & 0.0085 \\
\hline
\end{tabular}

\subsection{Results of Tech Mahindra Stock Price Prediction}

Over 3300 samples of Tech Mahindra were considered. Of those samples, 2800 samples were used in training and other samples were used for testing. Predictions are as shown in Figure 8 and 9. Even these graphs indicate the LSTM could predict better than compared to Standard Averaging.

\subsection{Results of WIPRO Stock Price Prediction}

Similar method was followed for WIPRO by considering nearly 5000 samples. Out of which 4000 samples was used for training and other samples were used for testing. Results of the prediction are as shown in Figure 10 and 11. MSE was reducing over the epochs which made accuracy of the predictions even better compared to Standard Averaging.

Mean Square Error of various companies is calculated for 30 epochs. The results of Epoch 1 and Epoch 30 are represented in Table 1.

From the tabulated values, it can be inferred that Mean Square Error is reducing as the number of Epochs increases. MSE loss is going down as the amount of the training increases. This indicates the LSTM model is learning stock prediction movement. The graphs clearly indicate LSTM performs better than Standard Averaging.

\section{CONCLUSION}

This paper establishes a LSTM model which is comprised of 3 LSTM layers along with linear regression. This model proves it predicts more accurately than Standard Averaging. The Recurrent Neural Network having memory cells stores data that is very appropriate and uses this for further calculation. This special type of neural network helps the model to learn better.

Researchers future work stresses on using the stock related news either from twitter or any other social media and use this analysis along with mid price to predict stock price. Since, only the past data may not be sufficient to predict stock price as the market is too volatile and even depend on current situations. This can even increase accuracy of the model. 


\section{REFERENCES}

Bhardwaja, Narayanb, Vanrajc, \& Maitreyee. (2015). Sentiment Analysis for Indian Stock Market Prediction Using Sensex and Nifty. In $4^{\text {th }}$ International Conference on Eco-friendly Computing and Communication Systems. Elsevier Publication.

Coyne, S., Madiraju, P., \& Coelho, J. (2017). Forecasting Stock Prices Using Social Media Analysis. IEEE 15th Intl Conf on Dependable, Autonomic and Secure Computing.

Ding, G., \& Qin, L. (2019). Study on the prediction of stock price based on the associated network model of LSTM. Int. J. Mach. Learn. \& Cyber. .10.1007/s13042-019-01041-1

Gao, , Chai, , \& Liu, . (2017). Applying long short term memory neural networks for predicting stock closing price. 8th IEEE International Conference on Software Engineering and Service Science (ICSESS).

Ghosh, , Bose, , Maji, , Debnath, , Sen, , \& Chowdhury, . (2019). Stock Price Prediction Using LSTM on Indian Share Market. Proceedings of 32nd International Conference on Computer Applications in Industry and Engineering.

Hamzaebi, C., Akay, D., \& Kutay, F. (2009). Comparison of direct and iterative artificial neural network forecast approaches in multi-periodic time series forecasting. Expert Systems with Applications, 36(2), 3839-3844. doi:10.1016/j.eswa.2008.02.042

Hochreiter, S., \& Schmidhuber, S. (1997). LSTM can solve hard long time lag problems. Advances in neural information processing systems. NIPS.

Ma, H., Ab, G. E., Menonab, V. K., \& Soman, , K.P. (2018). NSE Stock Market Prediction Using Deep-Learning Models. International Conference on Computational Intelligence and Data Science (ICCIDS 2018).

Menon, V. K., Vasireddy, N. C., Jami, S. A., Pedamallu, V. T. N., Sureshkumar, V., \& Soman, K. P. (2016). Bulk Price Forecasting Using Spark over NSE Data Set. International Conference on Data Mining and Big Data, 137-146. doi:10.1007/978-3-319-40973-3_13

Nivethitha. (2019). Future Stock Price Prediction using LSTM Machine Learning Algorithm. International Research Journal of Engineering and Technology, 6(3), 1182-1186.

Qiu, J., Wang, B., \& Zhou, C. (2020). Forecasting stock prices with long-short term memory neural network based on attention mechanism. PLoS ONE, 15(1). doi:10.1371/journal.pone.0227222

Sherstinsky, A. (2020). Fundamentals of Recurrent Neural Network (RNN) and Long Short-Term Memory (LSTM) network. Physica D: Nonlinear Phenomena, 404. 10.1016/j.physd.2019.132306

Zhang, G. P. (2003). Time-series forecasting using a hybrid ARIMA and neural network model. Neurocomputing, 50, 159-175. doi:10.1016/S0925-2312(01)00702-0

Zhang, J., \& Huang, M. L. (2013). 5Ws model for bigdata analysis and visualization. IEEE 16th International Conference on Computational Science and Engineering, 1021-1028. 